\title{
Évaluation de la qualité des plans d'eau urbains et périurbains : apport des approches transdisciplinaires et régionalisées
}

\author{
Veronica Mitroi $^{1}$, José-Frédéric Deroubaix ${ }^{2}$, Brigitte Vinçon-Leite ${ }^{3}$, Arnaud Catherine ${ }^{4}$, \\ Véronique Maleval ${ }^{5}$, Jean-François Humbert ${ }^{6}$ \\ 1 Sociologue, CNRS, UMR7618 Institut d'écologie et des sciences de l'environnement de Paris ( $i$ EES Paris), 75005 Paris, France \\ 2 Politiste, École des Ponts ParisTech, Laboratoire Eau environnement et systèmes urbains (LEESU), Champs-sur-Marne, \\ 77455 Marne-la-Vallée Cedex 2, France \\ 3 Biogéochimiste, École des Ponts ParisTech, Laboratoire Eau environnement et systèmes urbains (LEESU), Champs-sur-Marne, \\ 77455 Marne-la-Vallée Cedex 2, France \\ 4 Microbiologiste, MNHN, UMR7245 Molécules de communication et adaptation des microorganismes (MCAM), 75005 Paris, \\ France \\ 5 Géographe, Université de Limoges, UMR6042 Laboratoire de géographie physique et environnementale (GEOLAB), \\ 87036 Limoges, France \\ 6 Microbiologiste, INRA, UMR7618 Institut d'écologie et des sciences de l'environnement de Paris ( $i$ EES), 75005 Paris, France
}

Les plans d'eau urbains, mal connus et souvent perçus comme trop ordinaires ou artificiels, suscitent des attentes de la part des riverains, des usagers et des gestionnaires. De telles attentes qui renvoient à des lectures différentes des plans d'eau génèrent des tensions qui font de l'objectivation de leur état un enjeu cognitif fort. Cet article montre les difficultés rencontrées pour établir une telle caractérisation qui soit acceptable par les diverses parties prenantes. À la suite de plusieurs articles parus dans la revue sur les enjeux de production d'indicateurs d'état du milieu (NSS 1997, 5, 2 ; 2014, 22, 1), cette contribution met en avant la nécessité de prendre en compte aussi bien les points de vue des scientifiques que ceux des gestionnaires et des usagers. Ce faisant, elle questionne de manière tout à fait pertinente le rôle politique de la production de connaissances sur des objets d'apparence anodine, faisant progresser notre compréhension de l'interface science-société, sur des questions environnementales.

La Rédaction

\section{Mots-clés :}

recherche transdisciplinaire ; coconstruction des indicateurs de qualité plans d'eau urbains et périurbains ; concertation
Résumé - Produire des indicateurs de qualité des écosystèmes aquatiques anthropisés tenant compte des différents usages qui leur sont associés est un défi important qui ne peut être relevé qu'à l'interface de la recherche et de l'action. Dans cette optique, les auteurs portent un regard rétrospectif sur le travail mené pendant quatre ans au sein du programme de recherche PULSE afin d'évaluer la qualité des plans d'eau en Île-de-France. Après avoir montré l'intérêt d'étudier les plans d'eau périurbains, nous présentons comment le projet PULSE aborde ces objets hybrides, à mi-chemin entre « infrastructure urbaine » et « milieu naturel », à travers une approche transdisciplinaire centrée sur les usages de ces espaces. Nous discutons de la diversité des savoirs, des critères de valorisation de l'état de ces écosystèmes et des actions de gestion à travers lesquels les acteurs définissent la « qualité d'un plan d'eau ». Nous montrons ensuite jusqu'à quel point la transdisciplinarité permet d'imaginer des systèmes de caractérisation innovants et adaptés à la spécificité régionale de ces milieux et à leurs différents usages, tout en sachant que l'évaluation de ces systèmes n'échappe cependant pas à la nécessité, tant pour les scientifiques que pour les gestionnaires, de se référer à des critères de qualité universels et normatifs.

Auteur correspondant : V. Mitroi, veronicm@leesu.enpc.fr 


\author{
Keywords: \\ transdisciplinary \\ research; \\ coconstruction of \\ quality indicators; \\ institutional \\ arrangements; urban \\ water bodies; \\ consultation
}

\begin{abstract}
Assessing Urban and Peri-urban Pond Quality. Contribution of transdisciplinary and regionalized approaches. In order to consider the multiple uses associated with urban and peri-urban ponds, the production of quality indicators for these ecosystems needs to be conceived as an interface between research and action. PULSE the Peri-Urban Lakes, Society and Environment research program is developing a transdisciplinary and participatory approach to assess the quality of water bodies in the highly urbanized Île-de-France area. We show the interest of these water bodies, then discuss the diversity of knowledge, value criteria and management actions mobilized by the actors in order to define the "quality of urban water bodies". We then show how transdisciplinarity contributes to devising innovative characterization systems adapted to the regional specificity of these areas. These regional characterisation systems, however, do not exclude the need to refer to universal and normative quality criteria for both scientists and managers.
\end{abstract}

Occupant d'anciennes zones humides et des gravières, ou implantés simplement dans des zones propices d'un point de vue hydrographique, les plans d'eau urbains et périurbains de l'Île-de-France sont intégrés dans le tissu urbain selon différentes logiques d'aménagement et de gestion des eaux de surface. Ils accomplissent désormais des fonctions bien établies ou en cours de définition : épuration et stockage des eaux pluviales, services récréatifs (baignade, activités nautiques, etc.), conservation de la biodiversité, régulation climatique (îlots de fraîcheur), éléments de continuité écologique, paysage, etc. Bien qu'ils soient intégrés dans différents outils de gestion écologique (Natura 2000, Trame verte et bleue [TVB], Schéma d'aménagement et de gestion des eaux [SAGE], Schéma directeur de la région Île-deFrance [SDRIF]), les plans d'eau urbains sont des objets «ambigus » du point de vue de leur gouvernance, leur gestion étant le plus souvent dispersée entre des acteurs très divers : collectivités territoriales (services d'assainissement, espaces verts...), bases de loisirs, associations d'usagers et propriétaires privés.

Par ailleurs, ces écosystèmes demeurent marginaux dans les politiques de gestion de l'eau et notamment dans la directive-cadre européenne sur l'eau (DCE). Cette directive définit des objectifs de qualité des milieux aquatiques qui ne s'appliquent qu'aux seuls plans d'eau de plus de 50 ha, soit 10 en Île-de-France sur un total de 980 de plus de 5 ha. Pour les autres systèmes, les actions de gestion ou d'évaluation de leur qualité se concentrent généralement sur un usage ciblé et produisent des connaissances limitées aux usages définis par les gestionnaires.

En mettant au centre de sa réflexion la question de la multifonctionnalité et de la compatibilité des différents

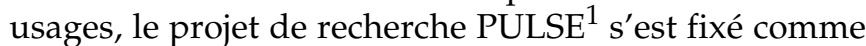
objectif de définir (i) une méthodologie de caractérisation

1 L'article est basé sur les résultats du projet PULSE (PeriUrban Lakes, Society and Environment), financé par le programme "Changementss environnementaux planétaires et sociétés » (CEPS) de l'Agence nationale de la recherche (ANR). Ce projet de 4 ans (2011-2014) réunissait 7 laboratoires globale et transdisciplinaire ${ }^{2}$ de la qualité des écosystèmes lentiques ${ }^{3}$ urbains et périurbains et (ii) des indicateurs de qualité de ces systèmes à disposition des acteurs en charge de leur gestion. Ainsi, à l'échelle régionale et à l'échelle locale, le projet interrogeait aussi bien les aspects écologiques que les usages sociaux et les pratiques de gestion des plans d'eau urbains. Le croisement des regards disciplinaires s'est accompagné d'une démarche de coconstruction, avec les gestionnaires, d'un référentiel partagé pour l'évaluation de la qualité des plans $\mathrm{d}$ 'eau de la région afin de guider ces derniers dans la formulation de leurs objectifs et des scénarios d'action. À travers cet objectif, le projet PULSE s'inscrit dans une forme de science régionalisée, inspirée de la philosophie des zones ateliers (Lévêque et al., 2000) et autres sites et plateformes expérimentales. Cependant, bien qu'assumant le postulat d'une spécificité territoriale des systèmes socionaturels, ce projet propose une méthodologie transposable à l'évaluation des milieux aquatiques présents au sein d'autres aires métropolitaines.

partenaires : 1'UMR7618 Biogéochimie et écologie des milieux continentaux (Bioemco) (devenue IEES Paris en 2014), 1'UMR7245 Molécules de communication et adaptation des microorganismes (MCAM), le laboratoire Eau environnement et systèmes urbains (LEESU), l'UMR8079 Écologie, systématique et évolution (ESE), l'UMR7190 Institut Jean le Rond d'Alembert, l'UMS3194 Centre de recherche en écologie expérimentale et prédictive-Écotron Île-de-France (CEREEP), 1'UMR6042 Laboratoire de géographie physique et environnementale (GEOLAB) et 1 laboratoire associé : 1'Unité de recherche sur l'écosystème prairial (UREP).

2 La recherche transdisciplinaire vise à produire des savoirs descriptifs, normatifs et orientés vers la pratique (Pohl et Hadorn, 2008) afin d'étudier des problèmes du monde vivant et du monde social.

3 Écosystèmes d'eaux calmes où les vitesses de l'eau sont lentes (lacs, étangs, mares, marécages, etc.), par opposition aux milieux d'eaux courantes qui correspondent aux écosystèmes lotiques. 


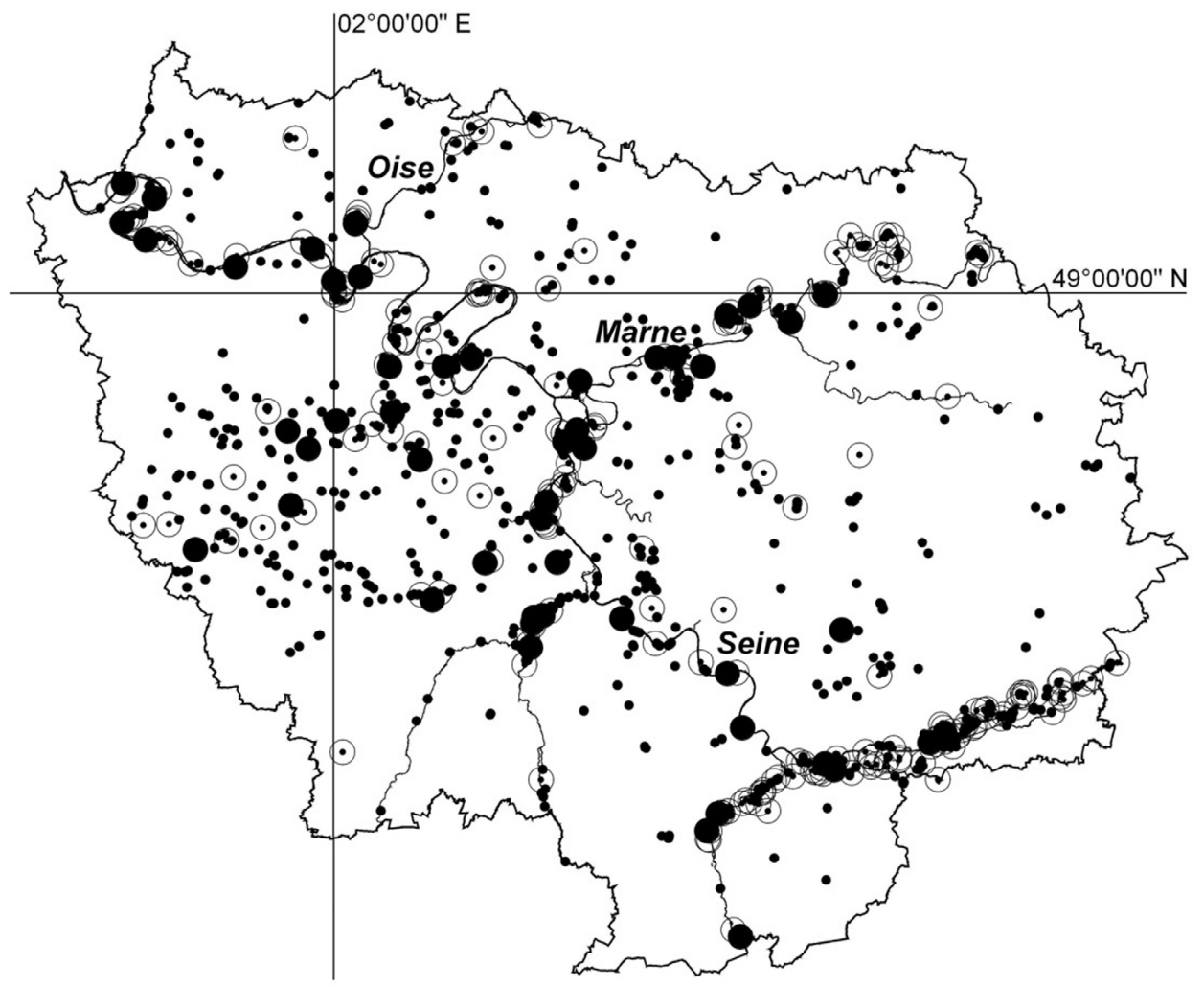

Fig. 1. Localisation des 980 plans d'eau d'Île-de-France (d'après Catherine et al., 2008). Chaque point correspond à un lac, les points cerclés représentent les plans d'eau de plus de 5 ha, les gros points les 49 lacs de plus de 5 ha étudiés dans le projet PULSE.

\section{Comment gérer la multifonctionnalité des plans d'eau de la région île-de-France?}

L'Île-de-France, région la plus peuplée et la plus urbanisée de France, bénéficie d'une richesse et d'une diversité de milieux aquatiques souvent mésestimées. En effet, celle-ci comprend environ 980 plans d' $\mathrm{eau}^{4}{ }^{4}$, dont environ 250 qui ont une surface supérieure à 5 ha (Catherine et al., 2008). Ces plans d'eau d'Île-de-France (Fig. 1) présentent une très grande diversité de taille, de connexion avec un bassin versant, d'état trophique, biologique et chimique, de statut juridique (privé, public ou communautaire) ou encore $\mathrm{d}^{\prime}$ usages. Soumis à des pressions anthropiques combinées et de diverses natures (pollutions urbaine, industrielle et agricole), ces écosystèmes représentent un vrai défi pour la compréhension de leur fonctionnement comme pour leur gestion.

$\mathrm{Au}$ sein des territoires métropolitains, la gouvernance des plans d'eau concerne principalement la préservation de la ressource et la gestion des risques d'inondation (Rankovic et al., 2012). À cela se sont ajoutées récemment

\footnotetext{
4 Par «plan d'eau» on entend toute surface d'eau permanente (définition donnée par la base de données Carthage de l'IGN). Le présent projet ne prend en compte que les plans d'eau de plus de 5 ha, une limite communément admise pour différencier un étang d'une mare.
}

des procédures de protection de la biodiversité ${ }^{5}$ et de valorisation des services écosystémiques (Amigues et Chevassus-au-Louis, 2011). C'est ainsi que certains documents de planification, notamment ceux relatifs à l'urbanisation du plateau de Saclay, prévoient la création de nouveaux étangs ou réseaux d'étangs pour faire face aux risques d'inondation, mais aussi pour améliorer le bienêtre des populations locales et préserver la biodiversité. De même, nombre de cartes dans le SDRIF rendent visibles ces masses d'eau en tant que « réservoirs de biodiversité » qui ont une place de premier ordre dans la mise en place des continuités écologiques (Mitroi et al., 2014). Sous l'effet des pressions politiques et sociales, il est donc exigé des plans d'eau situés en milieux urbains et périurbains de remplir une pluralité d'usages. Cependant, cette pluralité $\mathrm{d}$ 'usages requiert parfois des exigences de qualité peu compatibles ou peu réalistes au regard des contextes environnementaux locaux (baignades dans des sites fortement impactés par l'eutrophisation, par exemple).

Une situation issue du projet PULSE illustre bien ces conflits potentiels entre les différents usages et les

\footnotetext{
5 Sous la forme de réserves naturelles (parcs naturels régionaux, par exemple), de sites Natura 2000, de Zones naturelles d'intérêt écologique faunistique et floristique (ZNIEFF), de zones de protection spéciale (ZPS).
} 
fonctions d'un plan d'eau. Ainsi, sur l'étang de SaintQuentin-en-Yvelines, une "courbe consensuelle» des variations annuelles du niveau du plan d'eau a été définie afin de permettre conjointement la voile, la pêche, la gestion écologique et la gestion des débits. Les critères de qualité attachés aux usages sociaux peuvent rejoindre de façon ponctuelle les critères de qualité écologique. Ainsi, les sports nautiques et la baignade auront les mêmes exigences de qualité (basées sur la contamination par les bactéries fécales et les proliférations de cyanobactéries et compatibles avec les critères d'évaluation écologique). Cependant, dans les actions de gestion, besoins écologiques (variations des niveaux d'eau au profit des zones de nidification des oiseaux) et besoins sociaux (maintien d'un niveau élevé d'eau nécessaire pour la pratique de sports nautiques ou d'un niveau bas pour assurer le contrôle des ruissellements) entrent parfois en contradiction. L'enjeu majeur de la gestion de ces milieux est donc bien souvent la multifonctionnalité, l'articulation des compétences à déployer par les différents acteurs impliqués et l'exigence de faire coexister, dans un même espace, différents usages et donc différents critères de qualités. Une approche intégrée de caractérisation des logiques d'action et de gestion de ces milieux, prenant en compte leur état et les usages pouvant être potentiellement associés, reste donc à construire.

\section{Indicateurs de qualité et référentiels sociaux dans les pratiques d'évaluation - vers une interface science et action?}

La Gestion intégrée des ressources en eau (GIRE) s'est imposée ces dernières années comme le référentiel pour une gestion durable de l'eau et des milieux aquatiques. Cette approche devrait permettre de réconcilier l'ensemble des secteurs et groupes d'intérêt relatifs aux usages de l'eau (GWP, RIOB, 2009). Pour ce faire, elle encourage la participation des acteurs à tous les niveaux, de l'élaboration des textes juridiques à la définition des actions de gestion. En même temps, la GIRE mobilise un ensemble d'outils - évaluations sociales et environnementales, instruments économiques et systèmes d'information et de suivi - dont l'application aux « territoires aquatiques » relève d'une rationalité instrumentale. À contre-courant de ce parti pris techniciste, nous assumons ici que tout projet de gestion intégrée des plans $\mathrm{d}^{\prime}$ eau est avant tout un projet politique et nous proposons de restituer leur dimension sociopolitique aux indicateurs pouvant présider à sa définition et à sa mise en œuvre.

Toute tentative de construction d'indicateurs d'une gestion intégrée de ces masses d'eau suppose la constitution d'une communauté épistémique (Haas, 1992) capable de s'entendre sur des usages souhaitables de ces plans d'eau et sur la possibilité de les concilier. Cette communauté épistémique ne se réduit pas à un ensemble homogène $\mathrm{d}$ 'acteurs scientifiques, hydrologues ou écologues ne partageant pas les mêmes conventions dès lors qu'il s'agit, au sein de leur groupe professionnel, d'établir la « véracité » d'un fait ou d'un processus (Fernandez et al., 2011). La « coconstruction » $\mathrm{d}^{\prime}$ indicateurs dont il est ici question s'apparente largement à ce que Barbier et al. (2010), étudiant les mécanismes d'alerte et de gestion des pénuries d'eau - et reprenant l'expression de Jasanoff (2003) - qualifient de « science réglementaire». A contrario du modèle de gestion des milieux aquatiques de type « envirocratique ${ }^{6}$ » (Mermet et Barnaud, 1997), qui élude l'imbrication entre activités de production des savoirs et actions de gestion, la pratique d'une "science réglementaire » se réfère justement à la production des savoirs en tant qu'instruments de régulation. Cette science réglementaire est une activité fortement hybride : elle doit mêler des éléments de science hydrologique et d'écologie aquatique, des considérations relatives à la métrologie et aux données disponibles, des héritages de politiques $\mathrm{d}$ 'assainissement et de protection des espèces, des anticipations quant aux comportements des usagers.

Le modèle formel d'évaluation des modèles aquatiques par la DCE est fondé sur les notions de «bon état » écologique et de conditions de référence (Steyaert et Ollivier, 2007). Ces conditions de référence sont définies sur la base de processus d'intercalibration au niveau européen (Bouleau et Pont, 2014) et de compromis opérationnels. L'institutionnalisation de ce modèle va en partie à l'encontre de la logique d'hybridation des savoirs car il simplifie des concepts complexes relatifs aux processus écologiques et renvoie explicitement à l'idée qu'un écosystème est en «bon » ou en « mauvais » état, principalement en fonction de son statut trophique (i.e. niveau d'enrichissement en éléments nutritifs). Poussée à l'extrême, une telle vision incite à une homogénéisation de l'état trophique de ces milieux, l'état de faible enrichissement (i.e. oligotrophie) étant alors fortement valorisé. Cependant, du point de vue écologique, cette homogénéisation n'est pas souhaitable car elle pourrait conduire à une diminution de la diversité des systèmes écologiques au niveau régional et donc de la biodiversité qu'ils abritent.

De plus, les raccourcis opérationnels renvoient à l'idée que la qualité des milieux pourrait être nommée en dehors de tout référentiel social, quand en réalité un seul et même paramètre (par exemple la transparence de l'eau ou les concentrations en nutriments) aura une signification différente selon qu'on s'intéresse à l'état écologique

\footnotetext{
6 Dans ce modèle de gestion basé sur la rationalité de la science et sur celle de la gestion, il faut accorder les caractéristiques des milieux (décrits par des indicateurs scientifiques) avec les actions humaines (contrôlées par la gestion), en fonction des besoins et des problèmes identifiés par les sociétés.
} 


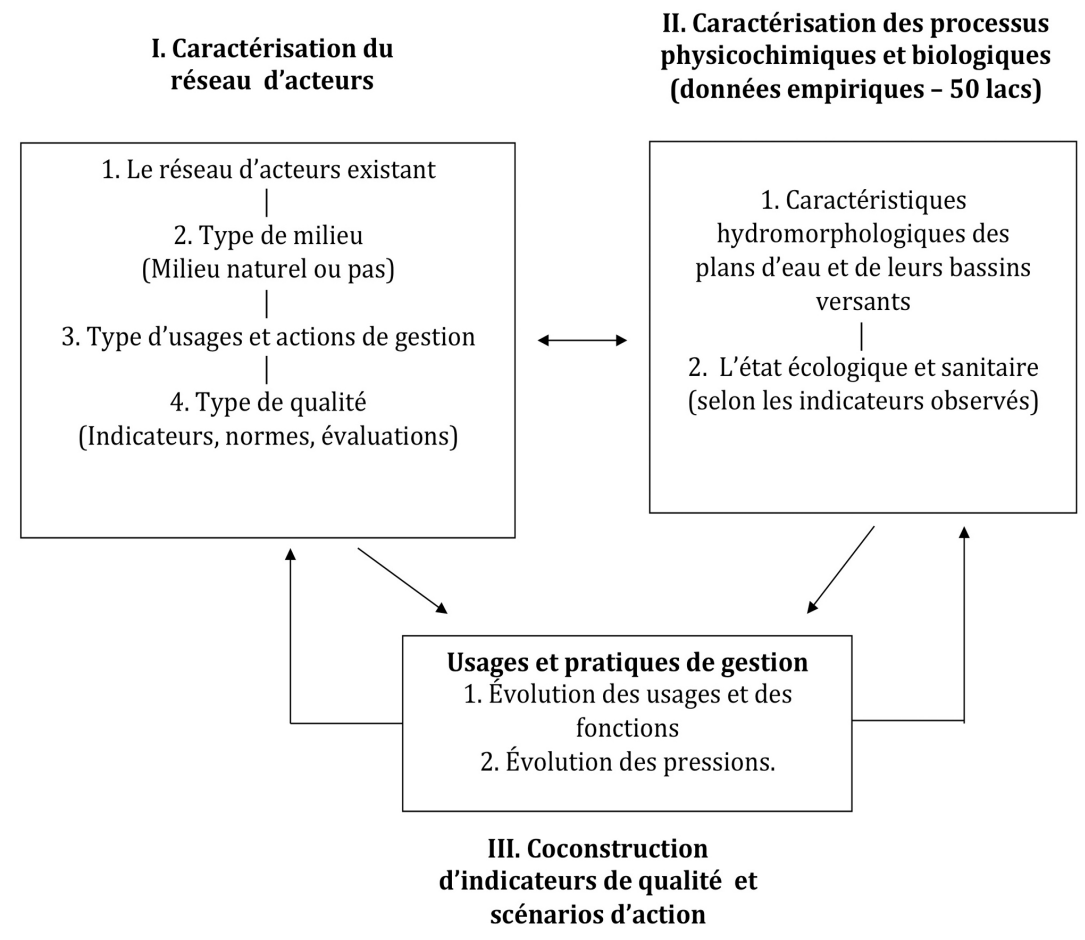

Fig. 2. Principales étapes de la démarche de recherche adoptée dans PULSE.

du plan d'eau ou à un usage particulier (par exemple la baignade). De manière générale, cette logique conduit à une survalorisation, de la part des politiques et des gestionnaires, de la biodiversité des milieux faiblement eutrophisés par rapport à d'autres processus écologiques (par exemple le cycle des nutriments, le stockage du carbone ou photosynthèse) pour le moins aussi importants du point de vue écologique mais aussi social.

Enfin, les scientifiques distinguent le plus souvent les questions relatives au bon état des écosystèmes de celles concernant l'état sanitaire, qui sont en lien direct avec certains usages (Lackey, 2001). Cette distinction s'illustre également au niveau réglementaire : la qualité écologique est réglementée au niveau européen (DCE), alors que la qualité sanitaire dépend de directives nationales (circulaires DGS/SD7A 2003/270, 2004/364, 2005/304) (Direction générale de la santé, 2003).

La définition d'indicateurs de qualité des plans d'eau constitue donc un processus de "bricolage ", impliquant la sélection, l'intégration et l'agrégation (Turnhout et al., 2007) de données, ainsi que la construction d'une norme (ou de valeurs seuils). De ce constat découle la nécessité $\mathrm{d}$ 'associer les gestionnaires à la définition d'indicateurs de qualité pertinents du point de vue des spécificités territoriales (hydrogéologie du plan d'eau et de son bassin versant, écologie de ses berges et de ses îles, occupation du sol sur le bassin versant, etc.) et plus largement des référentiels sociaux (pratiques des gestionnaires, usages, etc.).

\section{Le projet PULSE - un pari méthodologique de qualification des plans d'eau en tant qu'objets hybrides}

$C^{\prime}$ est dans le cadre de cette réflexion que les chercheurs du projet PULSE se sont proposés d'aborder ouvertement la dimension opérationnelle et sociopolitique des données qu'ils produisent, en interrogeant la place de ces données dans l'évaluation de la qualité de ces milieux et dans les processus de décision visant à améliorer leur « état socioécologique » (Fernandez et al., 2011 ; Lévêque et al., 2000 ; Lagadeuc et Chenorkian, 2009). Le projet se place ainsi dans une perspective transdisciplinaire, au croisement des regards portés par les sciences de la nature et par les sciences sociales, et en adoptant une posture propre à la sociologie des objets sociotechniques (Callon et Rip, 1992 ; Vinck, 2009). Nous considérons que les réseaux sociopolitiques impliqués dans la gestion de ces lacs déterminent, à la fois du point de vue cognitif (normes, indicateurs, etc.) et pratique (actions), le type de milieux (degré de «naturalité »), les critères de qualité reconnus, les modalités d'usage et les fonctions pouvant être développées. Cette hypothèse a guidé la structuration d'une démarche en trois grandes étapes (Fig. 2) : (I) la caractérisation du réseau d'acteurs et de leur conception des types de milieux, d'usages et de qualité des plans d'eau, (II) la caractérisation des processus physicochimiques et biologiques par les scientifiques et, au croisement de ces deux données, (III) la 
coconstruction d'indicateurs de qualité et la formulation de scénarios d'action.

Tout d'abord, l'état écologique et sanitaire de 49 lacs, considéré comme représentatif de la diversité des plans $\mathrm{d}^{\prime} \mathrm{eau}^{7}$ de la région Île-de-France, a été caractérisé grâce à des campagnes de mesures (2011-2013) réalisées sur une trentaine de paramètres ${ }^{8}$. Parallèlement, un questionnaire, renseigné par un ou plusieurs gestionnaires pour chaque plan d'eau, a permis de définir des variables dites « de gestion ». Les paramètres incluent notamment l'origine des plans d'eau, leur alimentation (par exemple par des nappes, des cours d'eau, des rejets pluviaux, des ruissellements), le temps de séjour de l'eau, les apports potentiels en matière organique et en polluants (dus, par exemple, à la présence de stations d'épuration, de collecteurs d'eau de pluie, de populations d'oiseaux), les usages présents et les pratiques d'entretien (curage, brassage, maîtrise de niveau d'eau, empoissonnement, par exemple). Ces variables ont permis de mieux prendre en compte les facteurs anthropiques qui influencent l'état écologique et sanitaire des lacs étudiés.

Les réseaux d'acteurs, leur expertise et leurs logiques d'action ont ensuite été analysés de façon plus détaillée par des études de cas sur trois lacs de la région parisienne (le lac de Créteil, le lac de Saint-Quentin-en-Yvelines et le lac d'Enghien-les-Bains). Les trois plans d'eau ont été retenus en croisant différents critères : formes urbaines (densité et forme du bâti), morphologie (taille, profondeur), connexion avec le système hydrographique et reconnaissance ou pas d'une valeur écologique. Deux campagnes d'enquêtes ont été réalisées, l'une auprès des gestionnaires ( 26 entretiens), l'autre auprès des usagers (94 questionnaires). Ces enquêtes ont permis de dégager les principales modalités permettant de formuler les enjeux de qualité en lien avec la gestion et les usages.

Enfin, les scientifiques impliqués dans le projet se proposaient de confronter les résultats de leurs travaux avec les pratiques et les attentes des gestionnaires. Il

\footnotetext{
7 Les 49 plans d'eau suivis pendant 3 ans ont été sélectionnés parmi les 248 plans d'eau de plus de 5 ha de la région et sont représentatifs de la diversité des milieux et des conditions environnementales locales (urbanité versus ruralité, par exemple) et hydrologiques (sur 49 plans d'eau, seule la moitié est connectée à un réseau hydrographique de surface) associées aux plans d'eau de la région Île-de-France (Catherine et al., 2008).

8 Ces paramètres sont représentatifs du fonctionnement physicochimique (température, $\mathrm{pH}$, conductivité, par exemple) et écologique (biomasse et diversité du phytoplancton, du zooplancton, etc.), de l'état microbiologique (densité en Escherichia coli et en entérocoques fécaux, structure génétique des communautés bactériennes, etc.) et de la contamination chimique (concentrations en hydrocarbures aromatiques polycycliques [HAP] et en métaux traces).
}

s'agissait principalement d'une démarche visant à rendre les savoirs scientifiques opérationnels, c'est-àdire à transformer les données en indicateurs (Vinck, 1994 ; Bouleau, 2006 ; Turnhout et al., 2007) mobilisables dans des actions concrètes de gestion. Un atelier participatif ayant pour but la coconstruction d'une grille d'évaluation, incluant les indicateurs considérés comme pertinents par les gestionnaires et appuyés par les données scientifiques, a été mis en place afin d'organiser ce transfert opérationnel des résultats vers la communauté des gestionnaires.

\section{Quelles pratiques en cours pour assurer la qualité des plans d'eau artificiels ?}

\section{La fragmentation des échelles et des compétences de gestion}

Les enquêtes réalisées auprès des gestionnaires nous ont permis d'identifier trois segments d'action et de compétences spécifiques : la compétence hydraulique (assurée par les services d'assainissement urbain), la compétence écologique (portée par les structures de protection de la nature) et la compétence sociale (en charge des aménagements et de l'organisation des usages sociaux). À chaque domaine de compétence correspond une logique (Fig. 3) qui associe des fonctions, des critères et des indicateurs de qualité spécifiques. Dans la logique écologique, soit une gestion des milieux principalement dédiée à la conservation de la biodiversité, les plans d'eau et leurs abords sont considérés comme des biotopes. Dans la logique infrastructurelle qui privilégie leur capacité de stockage hydraulique et la qualité physicochimique de l'eau, ils sont gérés en tant que milieux récepteurs. Dans la logique sociale, les gestionnaires (de bases de loisirs, par exemple) se focalisent sur les aspects liés aux usages tels que la qualité de l'eau (transparence, qualité microbiologique) mais aussi sur la biodiversité et les possibilités de nature en ville offertes par les plans d'eau.

Les indicateurs employés dans ces logiques ont différents degrés de formalisation et de normalisation qui renvoient à des ordres de grandeurs (biodiversité remarquable ou ordinaire), à des classements (mesures physicochimiques et bactériologiques), ou encore à des perceptions et des préférences liées aux usages sociaux (couleur, végétation et faune, transparence). Le " plan $\mathrm{d}^{\prime}$ eau » ne renvoie donc pas aux mêmes objets et aux mêmes échelles territoriales en fonction des acteurs et des usages qu'ils conçoivent. Il est considéré comme un objet tantôt lié aux masses d'eau de l'environnement proche, tantôt au territoire de vie des espèces qui y vivent, tantôt à l'aménagement urbanistique ou encore aux infrastructures urbaines d'assainissement. En effet, les plans d'eau 
Infrastructure urbaine Logique infrastructurelle

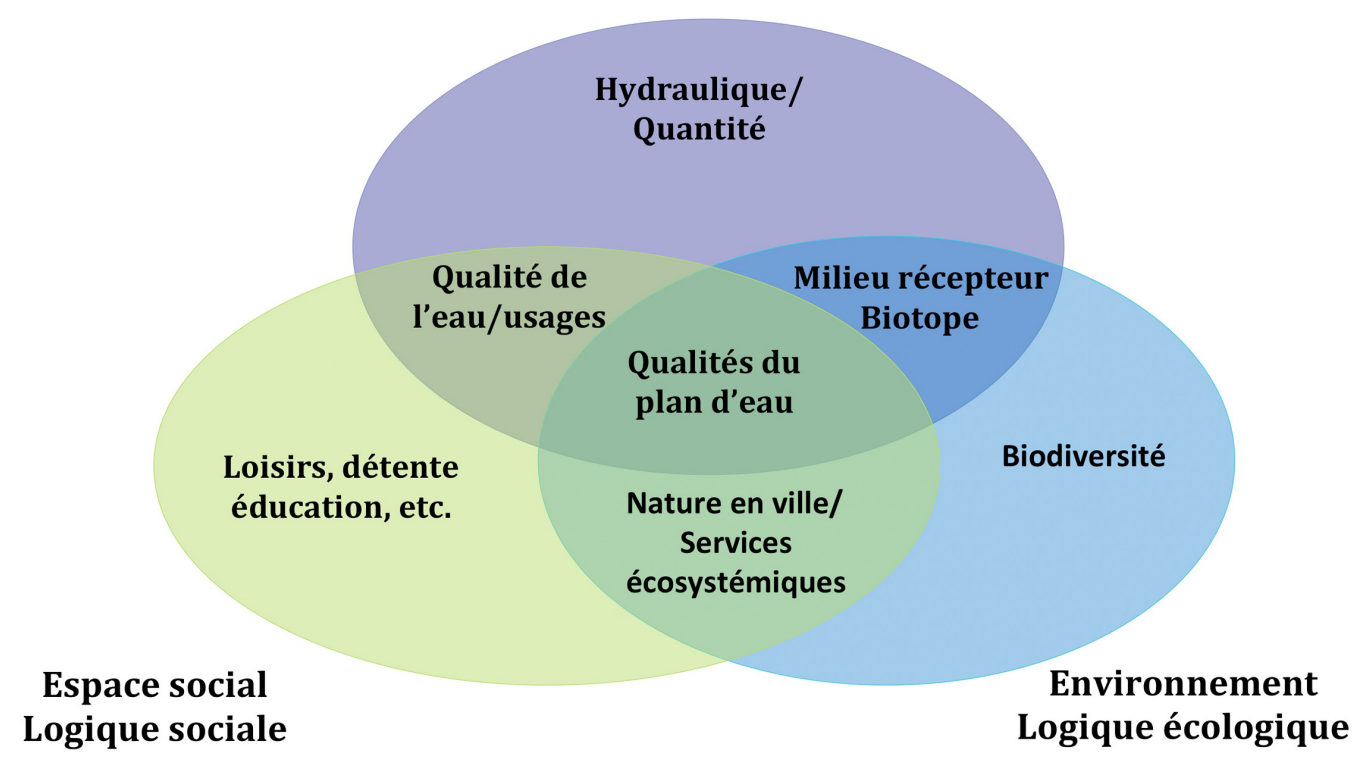

Fig. 3. Les dimensions de la qualité d'un plan d'eau urbain.

sont souvent dans le milieu urbain des infrastructures permettant la rétention des eaux pluviales et leur qualité dépend donc directement des réseaux d'assainissement qui collectent ces eaux ${ }^{9}$. Par ailleurs, la régulation hydraulique, réalisée au niveau départemental et qui influence également leur qualité, est prise en compte dans les schémas d'aménagement et de gestion des eaux (SAGE) qui doivent indiquer le débit maximum de rejet des eaux de ruissellement (dans les plans d'eau) et les débits de sortie dans le réseau hydrographique.

\section{Évolution des pratiques d'évaluation face aux nouvelles exigences réglementaires}

Si l'évaluation de la qualité sanitaire de l'eau (principalement selon des critères bactériologiques et chimiques) demeure un des piliers de la gestion des milieux aquatiques en France, l'émergence d'une évaluation globale de leur état écologique par rapport à un état de référence souhaitable représente une innovation récente en matière de politique de l'eau (Bouleau et Pont, 2014). Cependant, les gestionnaires des plans d'eau appliquent encore le plus souvent la logique de la grille dite « multi-usages » proposée par le ministère de l'Environnement dès les années 1970, « lisant » et « faisant » la

\footnotetext{
9 La gestion de ces réseaux dépend des mairies et des collectivités locales pour la collecte, des syndicats mixtes intercommunaux pour le transport et du SIAAP (Syndicat interdépartemental pour l'assainissement de l'agglomération parisienne) pour leur gestion à l'échelle interdépartementale.
}

qualité à un niveau local par rapport à un usage donné. Les informations fournies par les indicateurs de qualité sont directement traduites, là où il est possible de le faire, par des actions d'amélioration en tenant compte des contraintes d'usage. Cette logique et ces pratiques sont justement mises en question aujourd'hui par l'application de la DCE qui promeut une notion globale de qualité écologique des eaux de surface.

Bien que seuls dix plans d'eau en Île-de-France (i.e. ceux dont la surface est supérieure à 50 ha) soient concernés directement par les critères d'évaluation de la DCE, la généralisation de cette procédure a introduit des bouleversements dans les pratiques d'évaluation des collectivités locales ou des syndicats d'assainissement. En mettant l'accent sur le suivi des masses d'eau ${ }^{10}$ répertoriées, cette procédure conduit à négliger les plans d'eau qui ne rentrent pas dans le cadre du suivi DCE, même si ceux-ci faisaient pourtant l'objet d'une surveillance depuis plusieurs années. Ainsi, la Direction de l'eau et de l'assainissement du conseil départemental du Val-de-Marne nous indique à propos du lac de Créteil un exutoire d'eau pluviale ayant également des fonctions

\footnotetext{
10 La notion a été introduite en Europe par la DCE comme un terme générique faisant référence à tous les milieux aquatiques concernés par cette directive et dont les États membre s'engagent à dresser un état des lieux. En France, 33 types de masses d'eau regroupant des milieux aquatiques homogènes (cours d'eau, plans d'eau, eaux littorales, etc.) ont été définis du point de vue de certaines caractéristiques naturelles (relief, géologie, climat, débit...) (Arrêté du 12 janvier 2010, Article R.212-3 du Code de l'environnement).
} 
de base de loisirs nautiques : « Depuis 2010, notre réseau de suivi n'est plus subventionné par l'Agence de l'Eau, qui n'a retenu aucun plan d'eau de notre département, parce qu'ils ne sont pas dans les critères de la DCE. Le suivi que nous faisons sur le lac de Créteil est un suivi volontariste, sans aide financière. Il pourrait être arrêté à un moment donné si nous manquions de ressources».

D'autre part, se pose la question du statut juridique et administratif de ces exutoires d'eaux pluviales qui, bien que largement artificiels, sont très souvent connectés directement à des masses d'eau considérées comme «plus naturelles », à l'exemple des nappes phréatiques et des cours d'eau. Or, ce critère de continuité hydrologique est utilisé par la sphère politique pour justifier des objectifs de qualité pour les plans d'eau : «On part du principe qu'en se déversant dans la Seine, on aura les mêmes exigences de qualité que pour les masses d'eau identifiées dans les schémas directeurs d'aménagement et de gestion des eaux (SDAGE). On peut donc dire que $l^{\prime}$ on considère le lac [ $d^{\prime}$ Enghien] encore comme un milieu naturel. Il est dans un contexte hyperurbain, mais c'est encore [considéré] comme milieu naturel » (Service technique du Syndicat intercommunal d'assainissement de la région d'Enghien-les-Bains [SIARE], Val-d'Oise). Ainsi, l'identité de ces plans d'eau, au sein de leur bassin versant, se joue souvent en milieux urbains (i) en tant qu'objets de transition entre les services d'assainissement et les milieux naturels récepteurs et (ii) en tant qu'éléments dans la mise en place des continuités écologiques et des TVB (Mitroi et al., 2014). À ce titre, ces milieux devraient bénéficier du même type d'indicateurs écologiques (encore peu renseignés) ${ }^{11}$ que ceux retenus pour les milieux naturels (bio-indicateurs, par exemple).

Or, c'est dans le cadre des politiques de gestion hydraulique et des organismes qui gèrent les réseaux d'assainissement qu'est réalisé en général le suivi de la qualité des plans d'eau en Île-de-France, dans le but de déterminer leur impact sur les milieux récepteurs. C'est la raison pour laquelle parmi les critères retenus se retrouvent très souvent la qualité bactériologique ou les concentrations de matières en suspension. En fonction des résultats de ces suivis, les services d'assainissement prennent des décisions de maintenance ou d'investissement. Les critères d'évaluation choisis servent donc surtout à évaluer l'efficacité de leurs interventions sur les réseaux, et rarement le fonctionnement global du milieu qu'ils administrent. Dans cette optique opérationnelle, il est fréquent que les services décisionnaires regrettent la faible pertinence des études scientifiques au regard de leurs besoins de gestion : « On sait qu'il y a des dysfonctionnements sur

\footnotetext{
11 «Pour les plans d'eau, les éléments normatifs sont en cours d'élaboration, au niveau national et communautaire. Les objectifs sont actuellement fixés à dire d'expert » (Comité de bassin de l'Agence de l'eau Seine-Normandie, 2010, p. 21).
}

nos réseaux d'assainissement, nous n'avons pas forcément besoin d'études très chères, longues, pour prendre conscience qu'il faut agir » (Service technique Eau et assainissement, mairie de Créteil). En règle générale, le message des gestionnaires est assez clair : il faut simplifier et adapter les indicateurs de qualité aux usages et aux besoins d'action, parce que "de toute façon, dans l'eau, on trouve ce qu'on cherche! » (Service technique Eau et assainissement, mairie de Créteil).

\section{Croiser les regards sur la qualité des plans d'eau urbains : la mise en débat des indicateurs}

Dans quelles conditions les données produites par les scientifiques peuvent-elles être un support à la gestion afin de garantir les usages existants ou évaluer la pertinence de nouveaux usages? Quels indicateurs peuvent contribuer à la réflexion sur la multifonctionnalité ? Comment construit-on un référentiel territorial pour les plans d'eau d'Île-de-France ? Quels indicateurs seront appropriables par les gestionnaires ? Pour répondre à ces questions, nous avons débattu de la convergence et de la divergence entre données scientifiques et critères de qualité mobilisés par la gestion lors d'un atelier mixte réunissant 16 gestionnaires et représentants des usagers ${ }^{12}$ et 12 scientifiques du projet PULSE.

\section{Le lien manquant : une qualité écologique à définir à l'interface entre pratiques de recherche et de gestion}

L'atelier de coconstruction des indicateurs de qualité $\mathrm{s}^{\prime}$ est déroulé en plusieurs temps. La parole a tout d'abord été donnée aux gestionnaires, à qui l'on a demandé de nommer et de hiérarchiser les indicateurs de qualité qu'ils considèrent importants pour qualifier en général les milieux aquatiques urbains et périurbains et d'estimer ensuite (sur une échelle de 1 à 5) la valeur de ces indicateurs pour leurs propres plans d'eau. Leurs réponses ont ensuite été regroupées dans des catégories communes discutées et validées avec les participants. L'évaluation des plans d'eau par les participants à l'atelier présente un aspect contradictoire. En effet, alors qu'ils considèrent la

\footnotetext{
12 Ont été invités à participer à cet atelier tous les gestionnaires qui avaient été sollicités au cours du projet PULSE pour la réalisation des campagnes de mesures sur les 49 plans d'eau. Des représentants des institutions régionales ont également été invités. Le panel des personnes qui ont répondu à notre invitation s'est structuré comme suit : 7 agents travaillant dans le domaine des politiques de l'eau et des services d'assainissement, 7 agents dans celui de la gestion de la nature et 2 représentants des bases de loisirs et des associations d'usagers.
} 
qualité « globale » de leur plan d'eau comme étant relativement faible (indice 2,7), ils ont eu tendance à donner des notes supérieures aux indices spécifiques de qualité (qualité physicochimique, sanitaire, écologique). De manière intéressante, $l^{\prime}$ indice de qualité le mieux noté est « la qualité des usages sociaux » (indice 4), indice qu'ils considèrent comme le moins important pour juger de la qualité d'un plan d'eau. La qualité dite paysagère, qui concerne l'aménagement des berges et l'environnement immédiat, apparaît également en très bonne position. La fonction sociale des plans d'eau est donc considérée comme largement remplie, même si, aux yeux des gestionnaires, elle constitue la catégorie la moins importante pour juger de la qualité globale des plans d'eau. Cette dernière semble surtout déclassée par les qualités sanitaire (bactériologique) et physicochimique (nutriments, matière organique, polluants), paramètres sur lesquels les gestionnaires considèrent avoir peu de contrôle au niveau local.

Si la plupart d'entre eux montrent un intérêt particulier pour la qualité écologique des milieux aquatiques, nous avons pu constater qu'ils utilisent les indicateurs de qualité de l'eau (pollution, bactériologie, etc.) et les indicateurs écologiques (biodiversité, inventaire des espèces) de manière différenciée sans pour autant être capables de formuler clairement des correspondances entre eux. Les actions relatives à l'amélioration de la qualité de l'eau et celles concernant la conservation de la biodiversité ne se croisent pas forcément, et, sur le terrain, elles apparaissent même souvent contradictoires : «La finalité de la réserve, c'est la préservation des oiseaux, pas la préservation de la qualité de l'eau » (conservatrice de la réserve naturelle de Saint-Quentin-en-Yvelines). Dans une logique inverse, les responsables des bases de loisirs font remarquer qu'un nombre trop important d'oiseaux risque de poser problème pour la qualité bactériologique de l'eau et peut compromettre la baignade et les activités nautiques.

Les gestionnaires naturalistes (réserves naturelles et aires protégées) ont été surpris de ne pas trouver dans le cadre du projet PULSE de propositions d'indicateurs qui permettraient de prendre en compte la biodiversité sur et autour du plan d'eau. Cette absence est apparue comme une conséquence des moyens alloués au projet, des compétences disciplinaires spécifiques des chercheurs impliqués, mais s'explique surtout par le fait que scientifiques et gestionnaires ont une conception différente de la biodiversité. Certains scientifiques du projet PULSE ont ainsi fait valoir que le phytoplancton microscopique, descripteur biologique essentiel dans la littérature (Brucet et al., 2013), est tout aussi important pour juger de la qualité écologique d'un plan d'eau que de la biodiversité faunistique et floristique. Or, la logique des gestionnaires est essentiellement centrée sur la « biodiversité visible », qui constitue pour eux non seulement un enjeu de savoir mais aussi d'action. En effet, la présence d'espèces emblématiques permet une mobilisation accrue autour de la préservation des milieux.

Au-delà de ces contradictions entre différentes visions de la biodiversité, un accord a minima semble émerger sur la nécessité de penser les plans d'eau et leur environnement proche comme un même écosystème. Cependant, la question du périmètre de l'écosystème à considérer (la totalité du bassin versant ou simplement les berges) reste encore ouverte, car la «délimitation » $d$ 'un écosystème aquatique ne peut se concevoir en dehors d'une préoccupation scientifique ou politique particulière (Lackey, 2001). Un questionnement sur la qualité sanitaire n'implique pas le même « écosystème plan d'eau » qu'un questionnement sur les poissons, les oiseaux ou les macrophytes. De même, si le mode d'occupation du sol des bassins versants est considéré par de nombreux auteurs (Catherine $e t$ al., 2008) comme un indicateur majeur pour prédire l'état des milieux aquatiques récepteurs, il n'est pas pris en compte comme une donnée pertinente par les gestionnaires qui privilégient une logique de qualité environnementale des abords à une logique hydrologique de bassin versant.

\section{La construction des indicateurs de qualité : entre référentiel régional et normes globales}

La pertinence des indicateurs renseignés par les chercheurs a été discutée afin de définir le format d'une fiche synthétique d'évaluation permettant aux gestionnaires de disposer d'une image globale de l'état de leur plan d'eau en relation avec les 49 autres étudiés dans le projet PULSE. En agrégeant les mesures réalisées sur ces 49 lacs, les gestionnaires devaient disposer d'une lecture régionalisée leur permettant de situer la qualité de leur propre plan d'eau par rapport à un référentiel régional tenant compte des contraintes réelles de la région, liées notamment à l'urbanisation. Malgré l'intérêt manifeste des gestionnaires pour cette proposition, le besoin de se référer à des valeurs normées reconnues aux niveaux français et européen n'a pas pour autant été évacué.

Tout en étant conscients de la nécessité de se référer à ces indicateurs normés du type de ceux que propose la $\mathrm{DCE}$, les gestionnaires craignent que ces indicateurs globaux n'escamotent des indicateurs partiels et donnent des informations contradictoires : «Certains indicateurs globaux vous permettent de dire : la qualité est bonne alors que dans le même temps, on constate des mortalités de poissons ! » (représentant du parc naturel régional de la Haute Vallée de Chevreuse). Ainsi un indicateur global, bien que nécessaire pour se reporter à une norme ou à un référentiel plus général, serait techniquement moins pertinent qu'une série d'indicateurs « unidimensionnels » qui donneraient des informations plus ciblées et 
Tableau 1. Agrégation des indicateurs de qualité suivis dans le projet PULSE en indices de qualité.

\begin{tabular}{|c|c|}
\hline Indices de qualité & Indicateurs scientifiques \\
\hline \multirow{5}{*}{$\begin{array}{l}\text { Indice de qualité de } \\
\text { l'eau (IQE) }\end{array}$} & Concentration en oxygène dissous (mg/l) \\
\hline & $\mathrm{pH}$ \\
\hline & Conductivité $(\mu \mathrm{S} / \mathrm{cm})$ \\
\hline & Concentration en azote total $(\mathrm{mg} / \mathrm{l})$ \\
\hline & Concentration en phosphore total $(\mathrm{mg} / \mathrm{l})$ \\
\hline \multirow{4}{*}{$\begin{array}{l}\text { Indice } \\
\text { microbiologique } \\
\text { (IM) }\end{array}$} & $\begin{array}{l}\text { Concentration en chlorophylle-a }(\mathrm{mg} / \mathrm{l}) \text { : indicateur de } \\
\text { biomasse }\end{array}$ \\
\hline & $\begin{array}{l}\text { Pourcentage de cyanobactéries parmi le phytoplancton } \\
\text { (\%) : indicateur de micro-organismes potentiellement } \\
\text { toxiques }\end{array}$ \\
\hline & $\begin{array}{l}\text { Concentrations en microcystines }(\mu \mathrm{g} / \mathrm{L}) \text { : indicateur de } \\
\text { risque toxicologique }\end{array}$ \\
\hline & $\begin{array}{l}\text { Concentrations en } E \text {. coli et entérocoques intestinaux } \\
\text { (cellules } / \mathrm{mL} \text { ) : indicateur d'exposition aux } \\
\text { contaminateurs fécaux }\end{array}$ \\
\hline $\begin{array}{l}\text { Indice métaux } \\
\text { traces (IMT) }\end{array}$ & $\begin{array}{l}\text { Concentrations dissoutes de } 5 \text { métaux : le chrome, le } \\
\text { plombe, le zinc, le nickel et le cuivre ( } \mu \mathrm{g} / \mathrm{L})\end{array}$ \\
\hline $\begin{array}{l}\text { Indice } \\
\text { hydrocarbures } \\
\text { aromatiques } \\
\text { polycycliques (IHAP) }\end{array}$ & $\begin{array}{l}\text { Concentrations totales de } 5 \text { HAP : anthracène, } \\
\text { fluoranthène, benzo[a]pyrène, benzo[b]fluoranthène } \\
\text { Et benzo[g, } h \text {, i]perylène }(\mathrm{ng} / \mathrm{L})\end{array}$ \\
\hline
\end{tabular}

permettraient de mieux identifier les problèmes et d'orienter les actions. Malgré ces précautions, les gestionnaires restent demandeurs d'une «norme » ou d'une valeur-guide leur permettant de juger de façon plus formelle de la qualité de leurs plans d'eau et d'envisager des actions par rapport aux réglementations qui pourraient les concerner directement ou indirectement dans le futur.

Du côté des scientifiques, nous avons retrouvé ce même besoin de retenir un référentiel universellement partagé et validé par la communauté scientifique. Cependant, les critères partiels de qualité mentionnés par les gestionnaires ont été " traduits » par les scientifiques en termes d'indicateurs. Ils ont ensuite été agrégés en indices de qualité inspirés de la méthodologie proposée par le Programme des Nations unies pour l'environnement (PNUE) (Carr et Rickwood, 2008). Le résultat a conduit à la construction d'un indice global de qualité (IBQ) composé de 4 sous-indices : un indice de qualité de l'eau (IQE) ; un indice microbiologique (IM) ; un indice métaux traces (IMT) et un indice hydrocarbures aromatiques polycycliques (IHAP) (Tab.).

\section{Les représentations graphiques de la qualité relative des plans d'eau}

La présentation des données aux gestionnaires permet de réaliser une double lecture, à la fois locale et régionale. Une première représentation des indices de qualité leur donne la possibilité de visualiser les paramètres qui déclassent leur plan d'eau (Fig. 4) et l'ampleur de ce déclassement par rapport aux autres lacs de la région
(Fig. 5). Chaque indice est la moyenne des écarts à la valeur-guide pour les différents paramètres sélectionnés sur une échelle de 0 à 100 . Toute valeur d'un paramètre (qui correspond à la moyenne des valeurs observées sur les trois années) égale ou meilleure que la valeur-guide prendra la valeur 100 (i.e. bon état). La régionalisation de l'échelle s'effectue en attribuant, pour chaque paramètre, une note de 0 à la valeur correspondant à l'état le plus dégradé observé en Île-de-France. Ainsi, un indice ne peut prendre la valeur 100 que si l'ensemble des paramètres sélectionnés traduit un bon état du plan d'eau en question.

Les valeurs-guides ont été recherchées dans les normes du PNUE pour les indicateurs inclus dans l'indice de qualité de l'eau, dans les directives européennes relatives aux normes sanitaires ${ }^{13}$ pour les indicateurs de l'indice microbiologique, et dans les normes de qualité environnementale ${ }^{14}$ (NQE) définies par 1'Europe ou par le ministère de l'Écologie, du Développement durable et

\footnotetext{
${ }^{13}$ Les directives européennes 2006/7CE et 2014/101/UE (Commission européenne, 2014) et la circulaire produite par la Direction générale de la santé applicable aux zones de baignades et d'activités nautiques (circulaire DGS/SD7A 2003/ 270), à nouveau diffusée en 2004 (DGS/SD7A 2004/364) et en 2005 (DGS/SD7A 2005/304) basées sur les valeurs-guides de l'OMS.

14 Les valeurs-guides choisies correspondent aux normes de qualité environnementale (NQE) de la directive européenne 2013/39/UE du Parlement européen et du Conseil modifiant la directive 2000/60/CE (Parlement européen et Conseil de l'Union européenne, 2013).
} 


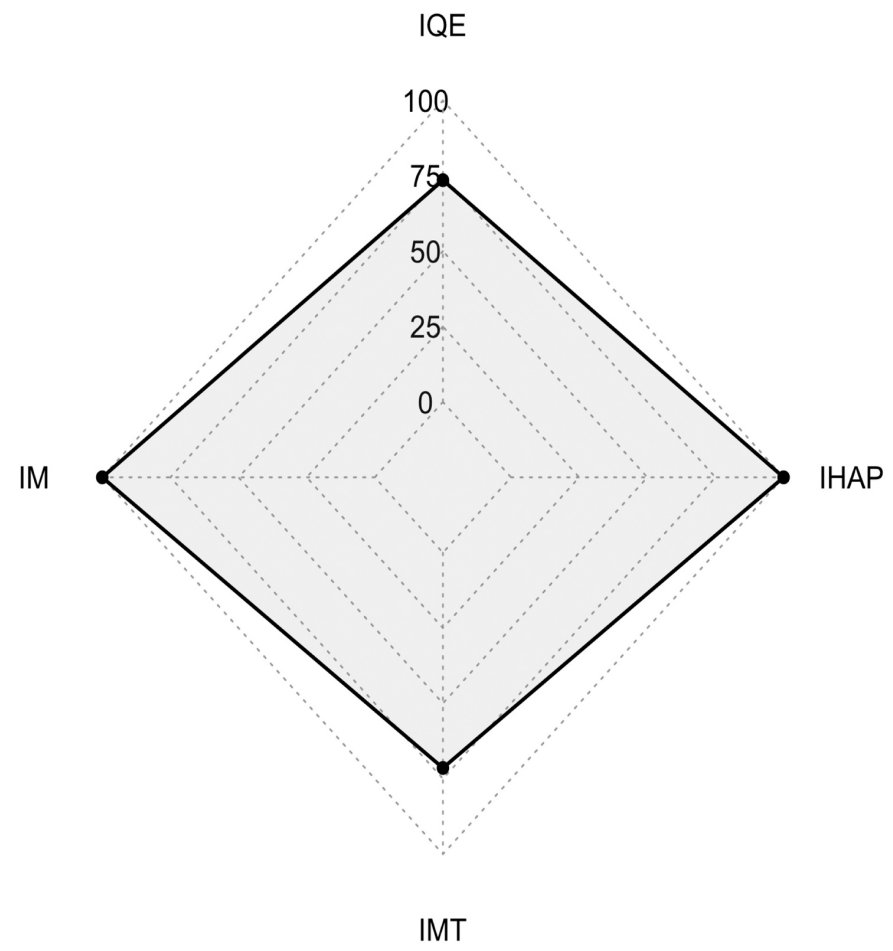

Fig. 4. Exemple de représentation graphique des indices de qualité caractérisant l'état écologique et sanitaire d'un plan d'eau (la valeur 100 indique un bon état écologique ; toute valeur inférieure à 100 correspond à un état dégradé).

de l'Énergie pour l'indice métaux traces et l'indice HAP. Ce choix en matière de valeurs-guides va dans le sens des demandes des gestionnaires, qui veulent pouvoir s'appuyer sur des référentiels validés par des tiers universels.

Une deuxième représentation (Fig. 5) permet d'observer le positionnement du plan d'eau pour chaque paramètre, dans le contexte des 49 lacs échantillonnés en région Île-de-France, à travers une distribution cumulative $^{15}$ (valeur moyenne des trois années et écart type associé).

Ces représentations simplifiées permettent d'avoir une image intuitive d'ensemble de chaque paramètre renseigné et des indices retenus. Les gestionnaires peuvent ainsi voir, tout à la fois, les paramètres déclassant leur plan d'eau par rapport à une norme globale, et leur position par rapport aux autres plans d'eau de la région. Les indicateurs ainsi produits peuvent servir de support à la décision et répondre, par exemple, au besoin de hiérarchiser les usages en fonction de la qualité du milieu au regard des exigences de multifonctionnalité : « Est-il raisonnable de vouloir faire une baignade dans un plan d'eau dépassant systématiquement les seuils de qualité liés à l'eutrophisation? » (chercheur PULSE) et à

\footnotetext{
15 Les lacs sont classés par ordre croissant pour chaque paramètre considéré.
}

l'inverse : « Est-il raisonnable d'interdire la baignade dans un plan d'eau où les conditions de la baignade sont remplies pour favoriser d'autres usages? » (chercheur PULSE). L'utilisation des indicateurs pour qualifier un plan d'eau et en définir les usages reste cependant un exercice éminemment politique, qui dépend des objectifs de gestion fixés par les acteurs : «La qualité voulue et recherchée est en fonction des objectifs de gestion » (représentant du parc naturel régional de la Haute Vallée de Chevreuse).

\section{Conclusion}

La production d'indicateurs utilisés dans l'évaluation et la surveillance de la qualité des milieux aquatiques s'impose comme une pratique incontournable afin d'assurer leur gestion durable (Deroubaix, 2008 ; Bouleau, 2012). Même les plans d'eau urbains et périurbains, moins concernés par les instances réglementaires qui imposent l'utilisation des indicateurs globaux de qualité, ne font pas exception à cette règle. Les gestionnaires concernés veulent avoir néanmoins la possibilité d'évaluer la qualité des masses d'eau dont ils ont la responsabilité en se reportant à des indicateurs normés afin de guider leurs actions et savoir ensuite si le résultat va «dans le bon sens » réglementaire. En même temps, ils 

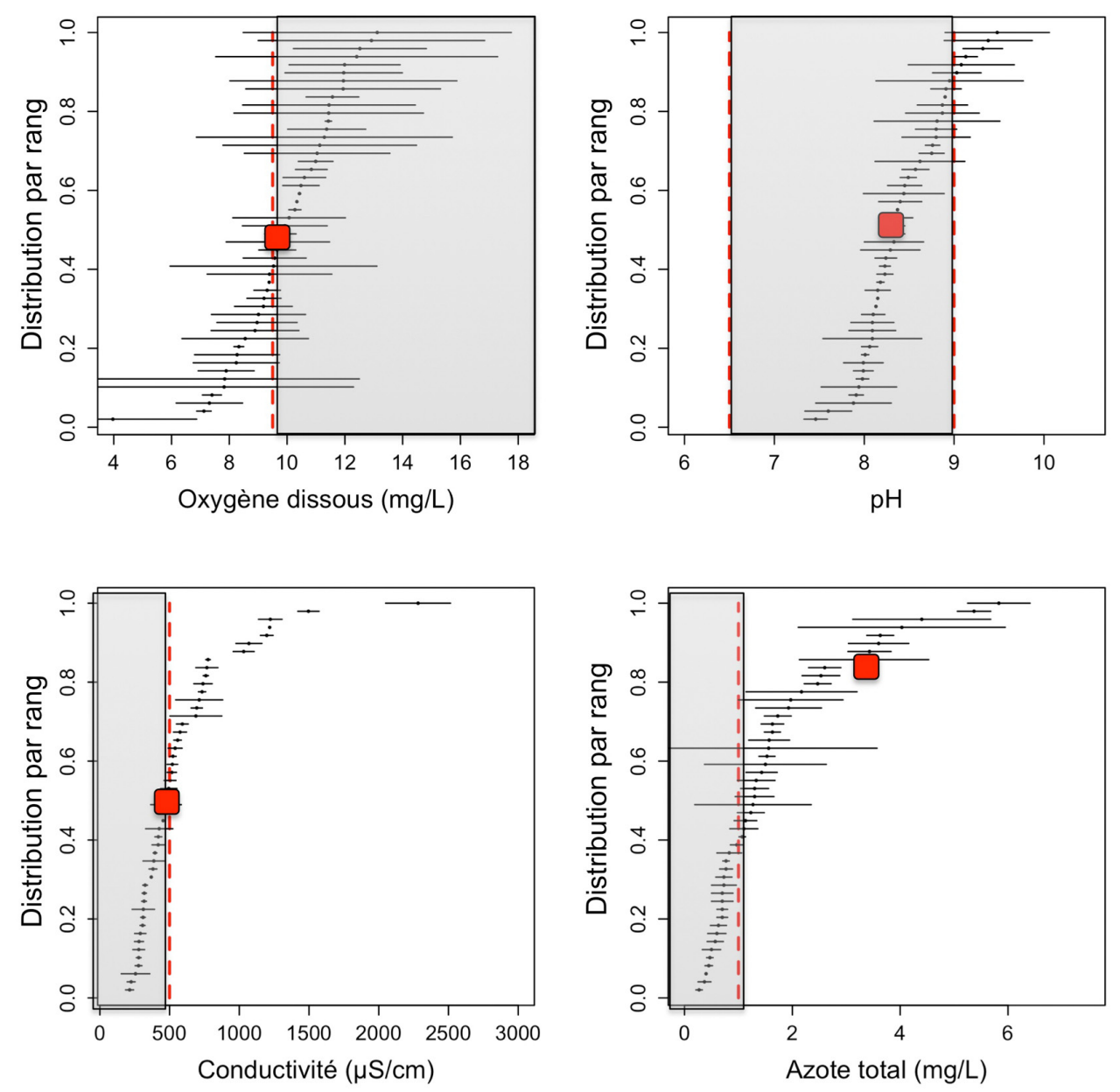

Fig. 5. Positionnement des écosystèmes dans un référentiel régional et normatif. La zone grisée correspond au bon état du plan d'eau. Chaque site est caractérisé par un segment dont les extrémités sont les valeurs maximale et minimale des trois années. Le plan d'eau considéré dans cette étude est représenté par un point rouge.

font état de l'intérêt d'un système d'indicateurs destiné à construire un référentiel régional de qualité de leur plan d'eau. Ce référentiel pourrait s'avérer utile dans la définition des priorités de gestion à l'échelle du territoire, certains plans d'eau privilégiant l'un ou l'autre des usages (biodiversité, usages récréatifs, etc.) en fonction de leurs spécificités géomorphologiques, physicochimiques, biologiques et écologiques. Ainsi, au lieu de contraindre les écosystèmes à des utilisations incompatibles avec leur niveau de qualité et qui demanderaient donc des investissements importants sans garantie d'atteindre le bon état requis, les gestionnaires sont conduits à s'interroger sur une organisation territoriale qui se dessinerait en fonction des critères de qualité actuelle ou potentielle des écosystèmes.

L'expérience menée dans le cadre du projet PULSE rappelle l'utilité du recours à des procédés flexibles de construction des indicateurs (Mermet et Barnaud, 1997), qui permet aux acteurs d'exprimer leurs besoins et leurs attentes et qui contribue ainsi à mieux accorder les capacités d'expertise disponibles aux actions de gestion. Cependant, cette expérience pointe également les limites d'une telle approche quand il s'agit de sélectionner dans des milieux aquatiques fortement anthropisés des indicateurs représentatifs aussi bien des processus écologiques et hydromorphologiques que des actions de gestion en lien avec les usages sociaux existants ou souhaités. Tandis que les indicateurs développés dans le cadre de la DCE témoignent d'une volonté de faire abstraction des usages existants, les gestionnaires impliqués dans PULSE s'efforcent, eux, de les préserver et de les valoriser. La mise en place de procédures participatives dans le cadre de la DCE et de la planification territoriale des masses d'eau (SDAGE et SAGE) ne permet de répondre que partiellement à cette complexité sociale. De nombreux auteurs (Fernandez et al., 2011 ; De Coninck et al., 2015) ont également montré les difficultés à dépasser les effets de cadrages que les indicateurs et les 
modèles écologiques induisent dans les procédures participatives parce que ces dernières sont largement orientées par les normes et les référentiels extraterritoriaux.

La tentative de socialisation et de territorialisation des données scientifiques (Lackey, 2001 ; Turnhout et al., 2007) par le biais du dispositif de recherche proposé dans le cadre du projet PULSE rencontre cependant certains obstacles. Pour les dépasser et réussir à fonder une GIRE adaptée aux spécificités régionales, il pourrait être judicieux de prendre le chemin inverse. Au lieu d'essayer de traduire les mesures scientifiques des paramètres a priori « importants » en termes d'indicateurs adaptés aux usages, les chercheurs et gestionnaires devraient partir des usages existants et chercher à « négocier » les indicateurs d'impact (nombre d'usagers et apports organiques associés à chaque type d'activité, par exemple) en fonction des contextes territoriaux. Associer chercheurs et gestionnaires à l'intégralité du processus de définition des indicateurs (critères, paramètres à mesurer, méthodes de mesure, etc.) pourrait faciliter la clarification des objectifs de gestion et la négociation des compromis entre les acteurs. Ces indicateurs assumeraient alors la fonction $d^{\prime}$ " " objets intermédiaires » (Vinck, 2009) permettant aux acteurs dotés d'intérêts et d'exigences de qualité très différents de se fixer des objectifs de gestion communs.

\section{Remerciements}

Les auteurs remercient les chercheurs impliqués dans le projet PULSE qui ont activement participé à la préparation de l'atelier et aux débats : Cécile Bernard (MNHN), Françoise Lucas (LEESU, Université Paris-EstCréteil-Val-de-Marne), Gérard Lacroix (IEES-Paris), ainsi que l'ensemble des gestionnaires qui nous ont accordé des entretiens ou qui ont collaboré à l'atelier mixte.

\section{Références}

Amigues J.-P., Chevassus-au-Louis B., 2011. Évaluer les services écologiques des milieux aquatiques: enjeux scientifiques, politiques et opérationnels, Vincennes, Onema., doi : http:// www.onema.fr/evaluer-les-services-ecologiques-desmilieux-aquatiques- 0 .

Barbier R., Riaux J., Barreteau O., 2010. Science réglementaire et démocratie technique. Réflexion à partir de la gestion des pénuries d'eau, Natures Sciences Sociétés, 18, 1, 14-23.

Bouleau G., 2006. Le débat sur la qualité de l'eau. Comment des données peuvent devenir des indicateurs ?, Ingénieries EAT, 47, 29-36.

Bouleau G., 2012. Ce que nous apprend l'histoire des indicateurs environnementaux, Revue forestière française, 5, 645-652.
Bouleau G., Pont D., 2014. Les conditions de référence de la directive cadre européenne sur l'eau face à la dynamique des hydrosystèmes et des usages, Natures Sciences Sociétés, 22, 1, 3-14, doi : http://dx.doi.org/10.1051/nss/2014016.

Brucet S., Poikane S., Lyche-Solheim A., Birk S., 2013. Biological assessment of European lakes: ecological rationale and human impacts, Freshwater Biology, 58, 6, 1106-1115.

Callon M., Rip A., 1992. Humains, non-humains, morale d'une coexistence, in Theys J., Kalaora B. (Eds), La terre outragée. Les experts sont formels!, Paris, Éditions Autrement, 140156.

Carr G.M., Rickwood C.J., 2008. Water quality: development of an index to assess country performance, United Nations Environment Programme Global Environment Monitoring System/Water Programme.

Catherine A., Troussellier M., Bernard C., 2008. Design and application of a stratified sampling strategy to study the regional distribution of cyanobacteria (Île-de-France, France), Water Research, 42, 20, 4989-5001.

Comité de bassin de l'Agence de l'eau Seine-Normandie, 2010. Le SDAGE 2000-2015. Du bassin de la Seine et des cours d'eau côtiers normands : pour un bon état des eaux en 2015, Nanterre, Agence de l'eau Seine-Normandie.

Commission européenne, 2014. Directive 2014/101/UE modifiant la directive 2000/60/CE du Parlement européen et du Conseil établissant un cadre pour une politique communautaire dans le domaine de l'eau (Texte présentant de l'intérêt pour l'EEE), 30 octobre.

De Coninck A., Deroubaix J.-F., Carré C., Becu N., Haghe J.-P., Hubert G., 2015. Confronter et recomposer les représentations pour concilier des politiques environnementales antagonistes. L'implication de la communauté scientifique dans la mise en débat de la continuité écologique sur le Grand Morin, in Béringuier P., Blot F., Desailly B., Saqalli M. (Eds), Environnement, politiques publiques et pratiques locales, Paris, L'Harmattan, 95-120.

Deroubaix J.-F., 2008. The co-production of a "relevant" expertise. Administrative and scientific cooperation in the French water policies elaboration and implementation since the 1990s, Hydrology and Earth System Sciences, 12, 4, 1165-1174.

Direction générale de la santé, 2003. Circulaire DGS/SD 7 A $n^{\circ}$ 2003-270 relative aux modalités d'évaluation et de gestion des risques sanitaires face à des situations de prolifération de microalgues (cyanobactéries) dans des eaux de zones de baignade et de loisirs nautiques, 4 juin.

Fernandez S., Bouleau G., Treyer S., 2011. Reconsidérer la prospective de l'eau en Europe dans ses dimensions politiques, Développement durable E territoires, 2, 3, doi : 10.4000/developpementdurable.9124.

GWP, RIOB (Global Water Partnership, [le partenariat mondial de l'eau], Réseau international des organismes de bassin), 2009. Manuel de gestion intégrée des ressources en eau par bassin, www.riob.org/IMG/pdf/GWP-RIOBManuelDeGIREparBassin.pdf.

Haas P.M., 1992. Introduction: epistemic communities and international policy coordination, International Organization, 46, 1,1-35. 
Jasanoff S., 2003. Technologies of humility: citizen participation in governing science, Minerva, 41, 3, 223-244.

Lackey R.T., 2001. Values, policy, and ecosystem health: options for resolving the many ecological policy issues we face depend on the concept of ecosystem health, but ecosystem health is based on controversial, value-based assumptions that masquerade as science, BioScience, 51, 6, 437-443.

Lagadeuc Y., Chenorkian R., 2009. Les systèmes socio-écologiques : vers une approche spatiale et temporelle, Natures Sciences Sociétés, 17, 2, 194-196.

Lévêque C., Pavé A., Abbadie L., Weill A., Vivien F.-D., 2000. Les zones ateliers, des dispositifs pour la recherche sur l'environnement et les anthroposystèmes, Natures Sciences Sociétés 8, 4, 44-52.

Mermet L., Barnaud G., 1997. Les systèmes de caractérisation des zones humides: construire l'expertise sous pression politique, Natures, Sciences, Sociétés, 5, 2, 31-40.

Mitroi V., De Coninck A., Vinçon-Leite B., Deroubaix J.-F., 2014. Establishing ecological and social continuities: new challenges to optimize urban watershed management, in Castellarin A., Ceola S., Toth E., Montanarin A. (Eds), Evolving water resources systems: understanding, predicting and managing water-society interactions. Proceedings of ICWRS2014, June, Bologna (Italy), Wallingford (U.K.), IAHS Press, 414-421.

Parlement européen et Conseil de l'Union européenne, 2000. Directive 2000/60/CE établissant un cadre pour une politique communautaire dans le domaine de l'eau, 23 octobre.
Parlement européen et Conseil de l'Union européenne, 2006. Directive 2006/7/CE concernant la gestion de la qualité des eaux de baignade et abrogeant la directive 76/160/CEE, 15 février.

Parlement européen et Conseil de l'Union européenne, 2013. Directive 2013/39/UE modifiant les directives 2000/60/CE et 2008/105/CE en ce qui concerne les substances prioritaires pour la politique dans le domaine de l'eau (Texte présentant de l'intérêt pour l'EEE), 12 août.

Pohl C., Hadorn G.H., 2008. Methodological challenges of transdisciplinary research, Natures, Sciences, Sociétés, 16, 2, 111-121.

Rankovic A., Pacteau C., Abbadie L., 2012. Services écosystémiques et adaptation urbaine interscalaire au changement climatique : un essai d'articulation, VertigO, Hors série 12, doi: 10.4000 /vertigo.11851.

Steyaert P., Ollivier G., 2007. The European water framework directive: how ecological assumptions frame technical and social change, Ecology and Society, 12, 1, http:// www.ecologyandsociety.org/vol12/iss1/art25/.

Turnhout E., Hisschemöller M., Eijsackers H., 2007. Ecological indicators: between the two fires of science and policy, Ecological Indicators, 7, 215-228.

Vinck D., 1994. La donnée: outil scientifique et outil de gestion, Environnement et société, 13, 75-87.

Vinck D., 2009. De l'objet intermédiaire à l'objet-frontière. Vers la prise en compte du travail d'équipement, Revue d'anthropologie des connaissances, 1, 3, 51-72.

Reçu le 10 octobre 2014. Accepté le 2 juin 2016 